\title{
Radiative transfer in one-dimensional inhomogeneous atmospheres ${ }^{\star}$
}

\author{
A. G. Nikoghossian ${ }^{1,2,3}$ \\ 1 Byurakan Astrophysical Observatory, 378433 Byurakan Aragatsotn, Armenia \\ e-mail: narthur@bao.sci.am \\ 2 Isaac Newton Institute of Chile, Armenian Branch, Armenia \\ 3 Research Institute for Astronomy and Astrophysics of Maragha, Iran
}

Received 18 March 2003 / Accepted 27 January 2004

\begin{abstract}
We use Ambartsumian's method of addition of layers to show that various problems, including the standard ones, of radiation transfer in a plane-parallel inhomogeneous atmosphere may be reduced to the solution of the Cauchy problems for linear differential equations. This allows avoiding the known difficulties arising in solving the boundary-value problems to which the classical approach leads. For the purpose of exposition, the paper deals with the simplest one-dimensional problem of multiple scattering for an atmosphere of finite optical thickness. The idea of the approach is that we start with determining the reflection and transmission coefficients of an atmosphere by solving the initial-value problem for a set of linear differential equations of the first order. After that the internal radiation field is found immediately without solving any new equation. The approach is applied to several classical problems of astrophysical interest. In particular, we evaluate the mean number of scatterings undergone by different types of photons. The transfer of radiation in an atmosphere with arbitrarily distributed internal sources is considered. Analytical solutions for these problems are obtained. Simple recursion formulas are derived to find the radiation intensity emitted by a multicomponent atmosphere. The problem of multiple scattering of radiation with partial redistribution over frequencies is discussed to demonstrate the generalization of the approach to the matrix case. The results of numerical calculations are given.
\end{abstract}

Key words. radiative transfer - methods: analytical - methods: numerical

\section{Introduction}

In many astrophysical problems, the necessity arises to interpret the radiation emitted by inhomogeneous media. The commonly used assumption of homogeneity simplifies the problem to a great extent and makes it possible to estimate some characteristics of the radiating medium averaged in some sense. However, the high-resolution observational data available nowadays afford an opportunity for a more detailed investigation of astrophysical objects and analysis of their radiation. This leads to pressure to develop a suitable theory of radiation transfer through an inhomogeneous atmosphere, providing new efficient methods of computations. Such attempts were made by a number of authors (see e.g., Jefferies \& Lindsey 1988; Gu et al. 1997; Cecchi-Pestellini \& Barletti 2001; Meinkohn \& Richling 2002; Juvela \& Padoan 2003) as well as by Nikoghossian et al. (1997, 1999); Nikoghossian \& Mouradian (2000). In this connection, the class of problems treating the transfer of radiation in atmosphere with varying single-scattering albedo is of special interest.

* All appendices are only available in electronic form at http://www. edpsciences.org
In this paper we shall limit ourselves to considering the one-dimensional version of these problems with a brief description of the possible generalizations. The analytical approach we apply is based on Ambartsumian's method of addition of layers. By introducing new quantities for describing the optical properties of a scattering atmosphere, the problem of diffuse reflection and transmission is reduced to the Cauchy problem for a linear system of first-order differential equations with variable coefficients. As a result, the requisite quantities are found for a family of atmospheres with different optical thickness. Further, we show that the internal radiation field is specified by the reflection and transmission capabilities of different parts of the atmosphere. Therefore the radiation field within the atmosphere is found directly without solving any new equation.

The linearity of the fundamental equations we obtain suggests a simple analytical procedure for the addition of layers. Having determined the reflectance and transmittance of an atmosphere, it is easy to find the other characteristics of the diffusion process such as the mean number of scatterings and the radiation field in atmospheres with arbitrarily distributed sources. These standard problems of astrophysical interest are reducible to the solution of the initial-value problems for the 
first-order differential equations. In some special cases, the problems are solvable analytically with solutions expressed in terms of elementary or special functions. Numerical schemes for the computational solution of these initial-value problems readily present themselves (see, e.g., Collatz 1960; Berezin \& Zhidkhov 1965; Bakhvalov 1973).

The outline of the paper is as follows. We begin, in the opening section of Sect. 2, by deriving differential equations for the reflection and transmission coefficients of inhomogeneous atmospheres. The polarity property of such an atmosphere is discussed. The following section is devoted to the problem of radiation transfer in an inhomogeneous atmosphere illuminated from outside. In Sect. 4 we consider the transfer of radiation in a multicomponent atmosphere, i.e. in an atmosphere with stepwise variation of the single-scattering albedo and of the power of the internal sources of energy. Recursion formulas are derived to determine both the external and internal radiation fields. The frequency-dependent problem of multiple scattering in an inhomogeneous atmosphere is treated in Sect. 5. The results obtained are discussed in the final section.

\section{Inhomogeneous atmosphere. The polarity property}

The method of addition of layers used in this paper was developed by Ambartsumian for homogeneous atmospheres (see Ambartsumian 1944, 1960; Sobolev 1963). The main idea underlying the method is to relate the reflectance $r$ and transmittance $q$ of an atmosphere to those of its individual parts. Referring the interested reader to the cited papers for details of the method, we restrict ourselves here to mentioning that it results in the pair of functional equations known as the summation laws for the reflectance and transmittance.

Note that the method of addition of layers is closely related to that of the invariance principle and invariant imbedding, therefore the same technique is named differently by different authors (see, e.g., Ambartsumian 1943, 1960; Sobolev 1963, 1974; Chandrasekhar 1960; Busbridge 1961; Bellman et al. 1956, 1960; Preisendorfer 1958, 1976; Abhyankar \& Fymat 1969, 1970; Peraiah 1984).

Our immediate goal in this section is the generalization of these results to the case of an inhomogeneous atmosphere in which the scattering coefficient $\lambda$ is allowed to vary with depth. It is natural to expect that now the reflecting and transmitting properties of the atmosphere will depend on the direction of the incident radiation. In other words, the atmosphere exhibits the so-called polarity property with respect to the sense of the incoming illumination, $-\mathrm{a}$ fact which has been brought out by Preisendorfer (1958). He concluded that this results in doubling the number of relevant equations.

Let us go further into this point. Since the symmetry of the problem is violated, it is logical to expect that we must have four equations (in twos for each boundary) to characterize the optical properties of the inhomogeneous atmosphere. In writing these, we have to distinguish the reflection and transmission processes due to a photon moving in one direction (say, to the boundary 0 in Fig. 1) from those related to a photon moving in the opposite direction. Let us agree to save notations $q$ and $r$

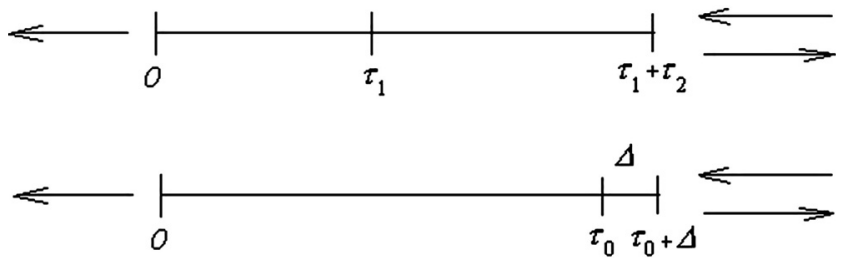

Fig. 1. A photon transfer process in an atmosphere consisting of two parts.

to denote the processes of the first kind, and introduce notations $\bar{q}$ and $\bar{r}$ to designate the processes of the second kind. All these quantities have a probability meaning and represent the probabilities that a photon will be transmitted or reflected from one or another boundary of the atmosphere. Note also that the transmission we discuss accounts for the single flight throughout of a medium. For a given function $\lambda$ of depth, the coefficients $r, \bar{r}$ and $q$ depend not only on optical thickness but also on the domain of variation of the function $\lambda(\tau)$. Bearing this in mind, let us consider an inhomogeneous atmosphere of optical thickness $\tau_{0}$ consisting of two parts with thickness $\tau_{1}$ and $\tau_{2}$, respectively (see Fig. 1). Then by virtue of the elementary laws of probability we may write

$$
\begin{aligned}
q\left(\tau_{1}+\tau_{2}\right)= & q\left(\tau_{1}\right) q^{*}\left(\tau_{2}\right) \\
& +q\left(\tau_{1}\right) r\left(\tau_{1}\right) \bar{r}^{*}\left(\tau_{2}\right) q^{*}\left(\tau_{2}\right)+\ldots \\
r\left(\tau_{1}+\tau_{2}\right)= & r^{*}\left(\tau_{2}\right)+\bar{q}^{*}\left(\tau_{2}\right) r\left(\tau_{1}\right) q^{*}\left(\tau_{2}\right) \\
& +\bar{q}^{*}\left(\tau_{2}\right) r^{2}\left(\tau_{1}\right) \bar{r}^{*}\left(\tau_{2}\right) q^{*}\left(\tau_{2}\right)+\ldots \\
\bar{q}\left(\tau_{1}+\tau_{2}\right)= & \bar{q}\left(\tau_{1}\right) \bar{q}^{*}\left(\tau_{2}\right) \\
& +\bar{q}^{*}\left(\tau_{2}\right) \bar{r}^{*}\left(\tau_{2}\right) r\left(\tau_{1}\right) \bar{q}\left(\tau_{1}\right)+\ldots
\end{aligned}
$$

$$
\begin{aligned}
& \bar{r}\left(\tau_{1}+\tau_{2}\right)=\bar{r}\left(\tau_{1}\right) \\
& \quad+\bar{q}\left(\tau_{1}\right) \bar{r}^{*}\left(\tau_{2}\right) q\left(\tau_{1}\right)+\bar{q}\left(\tau_{1}\right) r^{* 2}\left(\tau_{2}\right) \bar{r}\left(\tau_{1}\right) q\left(\tau_{1}\right)+\ldots,
\end{aligned}
$$

in which the asterisks denote the difference between domains of variation of $\lambda(\tau)$ in the two parts of the atmosphere. The infinite number of items in these sums is obviously due to the possibility of multiple interreflections between constituent parts.

Performing summations in Eqs. (1) and (2), we arrive at the following functional equations:

$$
\begin{aligned}
& q\left(\tau_{1}+\tau_{2}\right)=\frac{q\left(\tau_{1}\right) q^{*}\left(\tau_{2}\right)}{1-r\left(\tau_{1}\right) \bar{r}^{*}\left(\tau_{2}\right)} \\
& r\left(\tau_{1}+\tau_{2}\right)=r^{*}\left(\tau_{2}\right)+\frac{\bar{q}^{*}\left(\tau_{2}\right) r\left(\tau_{1}\right) q^{*}\left(\tau_{2}\right)}{1-r\left(\tau_{1}\right) \bar{r}^{*}\left(\tau_{2}\right)},
\end{aligned}
$$

which exhibit the semi-group properties of functions $q(\tau)$ and $r(\tau)$. One can easily check that the quantities $q$ and $\bar{q}$ satisfy the same equation with the same condition $q(0)=\bar{q}(0)=1$. Summation in Eq. (4) yields

$\bar{r}\left(\tau_{1}+\tau_{2}\right)=\bar{r}\left(\tau_{1}\right)+\frac{\bar{q}\left(\tau_{1}\right) \bar{r}^{*}\left(\tau_{1}\right) q\left(\tau_{1}\right)}{1-r\left(\tau_{1}\right) \bar{r}^{*}\left(\tau_{2}\right)}$.

Now let us replace $\tau_{1}$ by $\tau_{0}$, and $\tau_{2}$ by an infinitesimal layer $\Delta$. Specifically, the single-scattering albedo at the layer $\Delta$ is given 
by $\lambda=\lambda\left(\tau_{0}\right)$. It is remarkable that there is no need to specify the single-scattering albedo anywhere else other than at the boundary of the atmosphere. This is one of the salient traits of the method we use. The reflectance and transmittance of the layer $\Delta$ are

$r(\Delta)=\frac{\lambda\left(\tau_{0}\right)}{2} \Delta, \quad q(\Delta)=1-\left(1-\frac{\lambda\left(\tau_{0}\right)}{2}\right) \Delta$.

These relations are evidently independent of the side from which the layer $\Delta$ is illuminated. Passing to the limit as $\Delta \rightarrow 0$ leads to the following differential equations

$\frac{\mathrm{d} q}{\mathrm{~d} \tau_{0}}=-A\left(\tau_{0}\right) q\left(\tau_{0}\right)$,

$\frac{\mathrm{d} r}{\mathrm{~d} \tau_{0}}=\frac{\lambda\left(\tau_{0}\right)}{2}-\left[2-\lambda\left(\tau_{0}\right)\right] r\left(\tau_{0}\right)+\frac{\lambda\left(\tau_{0}\right)}{2} r^{2}\left(\tau_{0}\right)$,

$\frac{\mathrm{d} \bar{q}}{\mathrm{~d} \tau_{0}}=-A\left(\tau_{0}\right) \bar{q}\left(\tau_{0}\right)$,

$\frac{\mathrm{d} \bar{r}}{\mathrm{~d} \tau_{0}}=\frac{\lambda\left(\tau_{0}\right)}{2} q\left(\tau_{0}\right) \bar{q}\left(\tau_{0}\right)$,

where we introduced the notation $\boldsymbol{A}\left(\tau_{\mathbf{0}}\right)=\mathbf{1}-\frac{\lambda\left(\tau_{\mathbf{0}}\right)}{\mathbf{2}}\left[\mathbf{1}+\boldsymbol{r}\left(\tau_{\mathbf{0}}\right)\right]$. The initial conditions at $\tau_{0}=0$ are $q(0)=\bar{q}(0)=1, r(0)=$ $\bar{r}(0)=0$.

The forms of Eqs. (8) and (9) coincide with those for $\lambda=$ const. As could be expected, the functions $q\left(\tau_{0}\right)$ and $\bar{q}\left(\tau_{0}\right)$ are the solution of the same initial-value problem for the linear differential equation of the first order so that $q\left(\tau_{0}\right)=\bar{q}\left(\tau_{0}\right)$. This physically intelligible result is a direct consequence of the socalled reversibility principle of optical phenomena. Thus, the polarity of the one-dimensional atmosphere concerns merely the reflecting property, therefore the solution of Eqs. (8), (9) and (11) gives a complete idea of the reflecting and transmitting capacities for a set of inhomogeneous atmospheres of different optical thickness. We shall see in Sect. 5 that this result can be readily generalized to cover the frequency-dependent problem.

\section{The problem of diffuse reflection and transmission}

The nonlinear Eqs. (8), (9) and (11) obtained in the foregoing section make it possible to find the radiation emerging from the inhomogeneous atmospheres illuminated from outside. Note that the reflection coefficient $r\left(\tau_{0}\right)$ satisfies a separate equation of the Riccati type, the solution of which makes it possible to turn successively to Eqs. (8) and (11).

Now we shall show that Eqs. (8) and (9) can be reduced to a linear system of first-order differential equations for a certain properly chosen pair of functions. Let us define the functions $P\left(\tau_{0}\right)$ and $S\left(\tau_{0}\right)$ as follows

$P\left(\tau_{0}\right)=\frac{1}{q\left(\tau_{0}\right)}, \quad S\left(\tau_{0}\right)=\frac{r\left(\tau_{0}\right)}{q\left(\tau_{0}\right)}$.

Then Eq. (8) yields

$\frac{\mathrm{d} P}{\mathrm{~d} \tau_{0}}=\left(1-\frac{\lambda\left(\tau_{0}\right)}{2}\right) P\left(\tau_{0}\right)-\frac{\lambda\left(\tau_{0}\right)}{2} S\left(\tau_{0}\right)$,

which is the first of the requisite equations.
Now by virtue of Eq. (13) we have

$$
\begin{aligned}
& \frac{\mathrm{d} r}{\mathrm{~d} \tau_{0}}= \frac{\mathrm{d}}{\mathrm{d} \tau_{0}}\left(\frac{S}{P}\right)=\frac{1}{P} \frac{\mathrm{d} S}{\mathrm{~d} \tau_{0}}-\frac{S}{P^{2}} \frac{\mathrm{d} P}{\mathrm{~d} \tau_{0}}= \\
& \frac{1}{P} \frac{\mathrm{d} S}{\mathrm{~d} \tau_{0}}-\left(1-\frac{\lambda\left(\tau_{0}\right)}{2}\right) \frac{S\left(\tau_{0}\right)}{P\left(\tau_{0}\right)}+\frac{\lambda\left(\tau_{0}\right)}{2}\left(\frac{S\left(\tau_{0}\right)}{P\left(\tau_{0}\right)}\right)^{2} .
\end{aligned}
$$

On the other hand, Eq. (9) may be rewritten in terms of the functions $P$ and $S$ as

$$
\begin{aligned}
\frac{\mathrm{d} r}{\mathrm{~d} \tau_{0}}= & \frac{\lambda\left(\tau_{0}\right)}{2} \\
& -2\left(1-\frac{\lambda\left(\tau_{0}\right)}{2}\right) \frac{S\left(\tau_{0}\right)}{P\left(\tau_{0}\right)}+\frac{\lambda\left(\tau_{0}\right)}{2}\left(\frac{S\left(\tau_{0}\right)}{P\left(\tau_{0}\right)}\right)^{2} .
\end{aligned}
$$

Combining Eqs. (14) and (15) we finally obtain

$\frac{\mathrm{d} S}{\mathrm{~d} \tau_{0}}=\frac{\lambda\left(\tau_{0}\right)}{2} P\left(\tau_{0}\right)-\left(1-\frac{\lambda\left(\tau_{0}\right)}{2}\right) S\left(\tau_{0}\right)$.

Equations (13) and (16) represent the required linear system of differential equations. It must be supplemented by initial conditions $P(0)=1, S(0)=0$, which follow from the similar conditions for the functions $r\left(\tau_{0}\right)$ and $q\left(\tau_{0}\right)$. Having solved this system, the quantities $r\left(\tau_{0}\right)$ and $q\left(\tau_{0}\right)$ are readily found from Eq. (12). The system of Eqs. (13) and (16) plays an important role in the theory we put forward, since, as is shown in Appendix B, the determination of the internal radiation field is reduced to the same system of equations subject to proper initial conditions. As we shall see in Sect. 5, its generalization to the frequency-dependent case makes it possible to get rid of the troublesome procedure of matrix inversion.

This system may be put in a more compact vector-matrix form

$$
\frac{\mathrm{d} \boldsymbol{Y}}{\mathrm{d} \tau_{0}}=\boldsymbol{A} \boldsymbol{Y}, \quad \boldsymbol{Y}(0)=\left(\begin{array}{c}
1 \\
0
\end{array}\right)
$$

where

$$
\begin{array}{ll}
\boldsymbol{Y}\left(\tau_{0}\right) & =\left(\begin{array}{l}
P\left(\tau_{0}\right) \\
S\left(\tau_{0}\right)
\end{array}\right), \\
\boldsymbol{A}\left(\tau_{0}\right) & =\left(\begin{array}{ll}
1-\frac{\lambda\left(\tau_{0}\right)}{2} & -\frac{\lambda\left(\tau_{0}\right)}{2} \\
\frac{\lambda\left(\tau_{0}\right)}{2} & -\left[1-\frac{\lambda\left(\tau_{0}\right)}{2}\right]
\end{array}\right) .
\end{array}
$$

The problem we formulated is the classical initial-value problem for the system of first-order differential equations with variable coefficients. In some special cases it can be resolved in terms of elementary or special functions of analysis. On the other hand, there exist a number of computational schemes to obtain numerical solutions. In particular, if we wish to obtain the solution as a Taylor series in terms of powers of $\tau_{0}$, the following property of the matrix $\boldsymbol{A}$ will be helpful

$\boldsymbol{A}^{2}\left(\tau_{0}\right)=\left[1-\lambda\left(\tau_{0}\right)\right] \boldsymbol{I}$,

where $\boldsymbol{I}$ is the unit matrix.

Turning again to Eq. (17), we observe that, when $\lambda=$ const., the solution can be put into a form of the matrix exponential, which by virtue of Eq. (19) yields

$P\left(\tau_{0}\right)=\frac{D}{4 k}, \quad S\left(\tau_{0}\right)=\frac{1-k^{2}}{2 k} \sinh k \tau_{0}$, 
wherein $k=\sqrt{1-\lambda}$ and

$D=(1+k)^{2} \mathrm{e}^{k \tau_{0}}-(1-k)^{2} \mathrm{e}^{-k \tau_{0}}$.

Equations (20) and (21) lead to the well-known expressions for the transmittance and reflectance of a homogeneous atmosphere (see e.g., Sobolev 1963). Finally, if $\lambda\left(\tau_{0}\right)$ is differentiable, Eqs. (13) and (16) may be used to obtain separate differential equations for determining the functions $P$ and $S$. Differentiating the mentioned equations, after some simple transformations, we arrive at the following linear differential equations of the second order

$\frac{\mathrm{d}^{2} P}{\mathrm{~d} \tau_{0}^{2}}-\frac{\lambda^{\prime}}{\lambda} \frac{\mathrm{d} P}{\mathrm{~d} \tau_{0}}-\left(1-\lambda-\frac{\lambda^{\prime}}{\lambda}\right) P=0$,

$\frac{\mathrm{d}^{2} S}{\mathrm{~d} \tau_{0}^{2}}-\frac{\lambda^{\prime}}{\lambda} \frac{\mathrm{d} S}{\mathrm{~d} \tau_{0}}-\left(1-\lambda+\frac{\lambda^{\prime}}{\lambda}\right) S=0$,

subject respectively to the initial conditions

$$
\begin{aligned}
& P(0)=1, P^{\prime}(0)=1-\frac{\lambda(0)}{2} \\
& S(0)=0, S^{\prime}(0)=\frac{\lambda(0)}{2} .
\end{aligned}
$$

Two special cases of $\lambda\left(\tau_{0}\right)=\left[1+a \exp \left( \pm \tau_{0}\right)\right]^{-1}$ ( $a$ is a constant parameter) deserve particular attention and are treated in Appendix A. This is due to reduction of the order of Eqs. (22) and (23), which simplifies the solution of the problem. Besides, these two examples are rather instructive in understanding the specificity of radiation transfer in an inhomogeneous atmosphere, and will be used in further discussion.

Once the functions $P$ and $S$ are known for atmospheres of different optical thickness, the radiation field in them may be found directly without any new integration (Appendix B). In fact, the radiation intensities at any depth of the atmosphere are completely determined by the reflection and transmission capacities of its individual parts.

$$
U\left(\tau, \tau_{0}\right)=q\left(\tau_{0}\right) P(\tau) \quad V\left(\tau, \tau_{0}\right)=q\left(\tau_{0}\right) S(\tau),
$$

where $U$ and $V$ denote the probabilities to find the incident photon at depth $\tau$ moving respectively toward boundaries 0 and $\tau_{0}$.

Knowledge of the reflection and transmission coefficients makes it possible to solve some other problems of astrophysical interest concerned with the transfer of radiation in an inhomogeneous atmosphere. In Appendices C and D we evaluate respectively the mean number of scatterings undergone by photons of different kinds and the radiation field for an atmosphere with distributed sources of energy. Both of them are reduced to the easily solvable initial-value problems for the linear differential equations of the first order. In particular, for the intensities $I_{1}, I_{2}$, emerging from the boundaries $\tau_{0}$ and 0 of a self-radiating atmosphere, we obtain

$$
\begin{aligned}
& I_{1}\left(\tau_{0}\right)=\frac{1}{2} q\left(\tau_{0}\right) \int_{0}^{\tau_{0}}[1+r(t)] B(t) \frac{\mathrm{d} t}{q(t)}, \\
& I_{2}\left(\tau_{0}\right)=\frac{1}{2} \int_{0}^{\tau_{0}} q(t)\left[\lambda(t) I_{1}(t)+B(t)\right] \mathrm{d} t,
\end{aligned}
$$

where $B(\tau)$ denotes the power of distributed energy sources.

\section{Multicomponent atmosphere}

In astrophysics we frequently deal with radiating media with a complex fine structure. A number of exotic formations of solar chromospheric and coronal origin, such as prominences, streams, plums etc., are good examples. This leads to pressure to develop a suitable theory of radiative transfer through a multicomponent atmosphere, i.e., an atmosphere composed of a single type of structural elements differing in their optical and geometrical characteristics. The theoretical interpretation of radiation of such a medium encounters many difficulties of principle. The problem is simplified only in the LTE limit which is mostly realized for continua and some spectral lines. However, the formation of resonant and optically thick lines is controlled, in general, by multiple scattering effects which makes the theory complex. Allowance for random variations of the structural pattern needed in studying some non-stationary phenomena leads to further complications. Some analytical and numerical methods for solving the problems of radiation transfer in stochastic multicomponent atmospheres were proposed by Jefferies \& Lindsey (1988); Pomraning (1988); Lindsey \& Jefferies (1990); Gu et al. (1995); Barletti \& Cecchi-Pestellini (2001); Hegmann \& Kegel (2003). In a recent series of papers (Nikoghossian et al. 1997, 1999; Nikoghossian \& Mouradian 2000) we solved similar problems in interpreting the surface brightness fluctuations of solar prominences in the LTE and non-LTE lines. The situation where the structural elements are distinguished not only by their optical thickness and internal sources of energy but also by the value of the single-scattering albedo has not yet been handled, however.

The direct use of Eqs. (5) or their counterparts for a homogeneous atmosphere has a limited range of applications because of the non-linearity stipulated by the appearance of reflected beams in the course of multiple scattering. This leads, in particular, to complications in evaluating the mean statistical quantities characterizing the emission of a stochastic atmosphere since they are altered when a new component is added to the atmosphere. Only in some special model cases described in the above-mentioned series of our papers did we obtain closedform analytical expressions for these characteristics.

We shall see in this section that the approach proposed in this paper provides new possibilities for studying multicomponent non-LTE atmospheres. We start with considering the diffuse reflection and transmission problem for an atmosphere consisting of $N$ structural elements, each of which is described by its optical thickness, $\tau_{i}(i=1, \ldots N)$, and the value, $\lambda_{i}$, of the single-scattering albedo (Fig. 2). Let us agree to designate by $T_{m}$ the total optical thickness of $m$ elements (beginning from the first). Observing that $T_{N}=T_{N-1}+\tau_{N}$, we may use Eqs. (5) to write

$$
\begin{aligned}
& q\left(T_{N}\right)=\frac{q\left(T_{N-1}\right) q\left(\tau_{N}\right)}{1-r\left(T_{N-1}\right) r\left(\tau_{N}\right)}, \\
& r\left(T_{N}\right)=r\left(\tau_{N}\right)+\frac{r\left(T_{N-1}\right) q^{2}\left(\tau_{N}\right)}{1-r\left(T_{N-1}\right) r\left(\tau_{N}\right)},
\end{aligned}
$$

in which the bars are omitted in view of the homogeneity of the single element. 


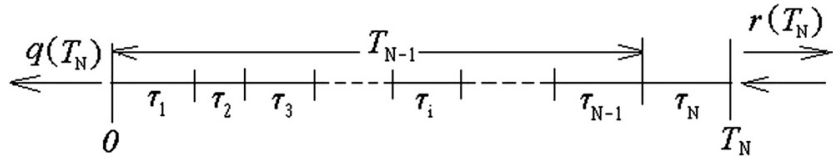

Fig. 2. The photon transfer through a non-LTE multicomponent atmosphere. Each of the structural elements is distinguished by optical thickness and the value of the single-scattering albedo.

Equation (28) may be rewritten in the form

$P\left(T_{N}\right)=a\left(\tau_{N}\right) P\left(T_{N-1}\right)-b\left(\tau_{N}\right) S\left(T_{N-1}\right)$,

where we introduced the quantities $a\left(\tau_{N}\right)=P\left(\tau_{N}\right), b\left(\tau_{N}\right)=$ $S\left(\tau_{N}\right)$, which are given by Eq. (20), with $\tau_{N}$ and $\lambda_{N}$ taken instead of $\tau_{0}$ and $\lambda$. Further, dividing Eq. (29) by Eq. (28), after some simple transformations we find

$S\left(T_{N}\right)=b\left(\tau_{N}\right) P\left(T_{N-1}\right)+c\left(\tau_{N}\right) S\left(T_{N-1}\right)$,

where

$$
\begin{aligned}
c\left(\tau_{N}\right) & =\frac{1-b^{2}\left(\tau_{N}\right)}{a\left(\tau_{N}\right)} \\
& =\frac{1}{4 k_{N}}\left[\left(1+k_{N}\right)^{2} \mathrm{e}^{-k_{N} \tau_{N}}-\left(1-k_{N}\right)^{2} \mathrm{e}^{k_{N} \tau_{N}}\right],
\end{aligned}
$$

with $k_{N}=\sqrt{1-\lambda_{N}}$.

Equations (30) and (31) with conditions $P\left(T_{0}\right)=1$, $S\left(T_{0}\right)=0$ represent the recurrence formulas, which readily allow one to determine the reflection and transmission capacities for any kind of multicomponent atmosphere. The main advantage of these equations is their linearity which is especially important in treating atmospheres with randomly varying optical characteristics. Actually, for a given distribution law of random parameters, this property makes it possible to average the global characteristics, $P, S$, of the multicomponent atmosphere for any number of structural elements. Thus, one may write

$\left\langle P\left(T_{N}\right)\right\rangle=\left\langle a\left(\tau_{N}\right)\right\rangle\left\langle P\left(T_{N-1}\right)\right\rangle-\left\langle b\left(\tau_{N}\right)\right\rangle\left\langle S\left(T_{N-1}\right)\right\rangle$,

$\left\langle S\left(T_{N}\right)\right\rangle=\left\langle b\left(\tau_{N}\right)\right\rangle\left\langle P\left(T_{N-1}\right)\right\rangle+\left\langle c\left(\tau_{N}\right)\right\rangle\left\langle S\left(T_{N-1}\right)\right\rangle$,

with $\left\langle P\left(T_{0}\right)\right\rangle=1,\left\langle S\left(T_{0}\right)\right\rangle=0$. The second moments are found in a similar manner. In turn, this allows us to study the dispersion of the optical properties of a multicomponent atmosphere with randomly varying parameter $\lambda$.

A procedure analogous to that above may be employed when obtaining the reflectance of the atmosphere if it is illuminated from the boundary 0. Departing from Eq. (6), we arrive at

$\bar{r}\left(T_{N}\right)=\bar{r}\left(T_{N-1}\right)+\frac{\bar{r}\left(\tau_{N}\right) q^{2}\left(T_{N-1}\right)}{1-r\left(T_{N-1}\right) \bar{r}\left(\tau_{N}\right)}$,

the counterpart of Eq. (29) for the case under consideration. Dividing Eq. (35) by Eq. (28) and introducing the notation $\bar{S}=$ $\bar{r} / q$, we finally obtain

$\bar{S}\left(T_{N}\right)=\frac{1}{P\left(T_{N-1}\right)}\left[P\left(T_{N}\right) \bar{S}\left(T_{N-1}\right)+b\left(\tau_{N}\right)\right]$.

We see that the requisite quantity $\bar{S}\left(T_{N}\right)$ is evaluated recurrently parallel to $P\left(T_{N}\right)$.
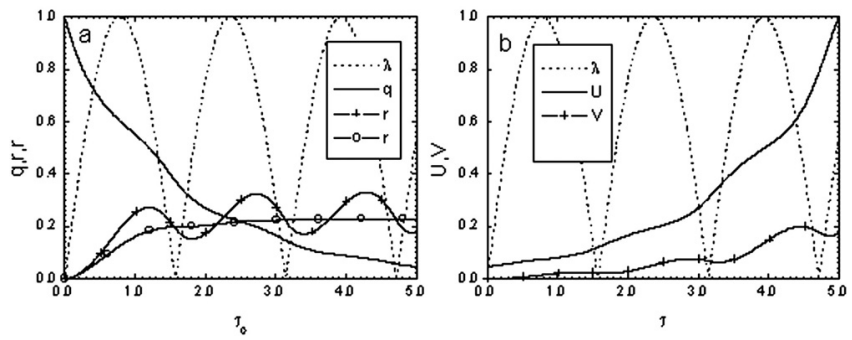

Fig. 3. Atmosphere with $\lambda(\tau)=|\sin 2 \tau|$ (dotted lines). a) Dependence of the transmittance $(q)$ and reflectances $(r, \bar{r})$ on optical thickness. b) Internal radiation field $(U, V$, see Appendix $\mathrm{B})$.

It should be emphasized that letting $\tau_{i}$ be equal and sufficiently small, we may consider Eqs. (30) and (31) as a rather efficient computational scheme for solving the fundamental system of Eqs. (13) and (16). Use of Eqs. (30) and (31) is especially recommended for solving the transfer problems with oscillating behavior of $\lambda$, or when $\lambda$ is approximated by functions with spikes. Such an example is displayed in Fig. 3. The left-hand panel shows the dependence of both reflectance and the transmittance on optical thickness for an atmosphere with $\lambda(\tau)=|\sin 2 \tau|$. The internal field of radiation in the atmosphere is shown in Fig. $3 b$.

Note in conclusion that one may use Eqs. (D.6) and (D.7) to derive the recursion formulas for determining the intensities of radiation emerging from a multicomponent atmosphere containing energy sources. To this end, we, again, consider an atmosphere consisting of $N$ structural elements, each of which is now characterized not only by optical thickness $\left(\tau_{i}\right)$ and the value of the single-scattering albedo $\left(\lambda_{i}\right)$, but also by the power of the internal sources of energy $\left(B_{i}\right)$. We assume that $\lambda_{i}$ and $B_{i}$ are constant within each component. Since $T_{N}=T_{N-1}+\tau_{N}$, we employ Eqs. (D.6) and (D.7) to obtain

$S_{1}^{*}\left(T_{N}\right)=S_{1}^{*}\left(T_{N-1}\right)+F\left(\tau_{N}\right)\left[S\left(T_{N-1}\right)+P\left(T_{N}\right)\right]$,

$$
\begin{aligned}
S_{2}^{*}\left(T_{N}\right)= & \frac{P\left(T_{N}\right)}{P\left(T_{N-1}\right)} S_{2}^{*}\left(T_{N-1}\right) \\
& +b\left(\tau_{N}\right) P\left(T_{N-1}\right) S_{1}^{*}\left(T_{N-1}\right)+a\left(\tau_{N}\right) F\left(\tau_{N}\right),
\end{aligned}
$$

where we have introduced the following notations: $S_{1}^{*}=I_{1} / q$, $S_{2}^{*}=I_{2} / q, F\left(\tau_{N}\right)$ is the intensity radiated to each side by the homogeneous layer $\tau_{N}$. The latter is given by (see Sobolev 1963)

$F\left(\tau_{N}\right)=\frac{B_{N}}{1-k_{N}^{2}} \int_{0}^{\tau_{N}} p\left(\tau, \tau_{N}\right) \mathrm{d} \tau$,

where

$$
\begin{aligned}
p\left(\tau, \tau_{N}\right)= & \frac{1-k_{N}^{2}}{4 k_{N} a\left(\tau_{N}\right)} \\
& \times\left[\left(1+k_{N}\right) \mathrm{e}^{k_{N}\left(\tau_{N}-\tau\right)}-\left(1-k_{N}\right) \mathrm{e}^{-k_{N}\left(\tau_{N}-\tau\right)}\right]
\end{aligned}
$$

is the photon exit probability. Performing an integration in Eq. (39) we finally obtain

$$
\begin{aligned}
F\left(\tau_{N}\right)= & \frac{B_{N}}{4 k_{N}^{2} a\left(\tau_{N}\right)} \\
& \times\left[\left(1+k_{N}\right) \mathrm{e}^{k_{N} \tau_{N}}+\left(1-k_{N}\right) \mathrm{e}^{-k_{N} \tau_{N}}-2\right] .
\end{aligned}
$$


Equations (37)-(39) are the required equations to find the intensities of radiation emerging from a multicomponent atmosphere with energy sources.

\section{Spectral line profiles}

The method developed in this paper may be easily generalized to encompass the frequency-dependent problems of radiative transfer. Furthermore, reasoning similar to that below makes it possible to handle three-dimensional problems.

This section intends to evaluate the profiles of spectral lines formed in an inhomogeneous atmosphere under the assumption that multiple scattering occurs in the atmosphere with redistribution over frequencies. We introduce the function $R\left(x^{\prime}, x\right)$ to denote the frequency redistribution law averaged over directions, where $x$ is the so-called dimensionless frequency which represents the frequency displacement from the center of the line measured in units of Doppler widths. In the case of completely noncoherent scattering, the redistribution function is factorized to yield $R\left(x^{\prime}, x\right)=A \alpha\left(x^{\prime}\right) \alpha(x)$, where $A$ is a certain constant and $\alpha(x)$ is the non-normalized profile of the absorption coefficient. We shall be restricted by treating the Doppler redistribution over frequencies when $A=1 / \sqrt{\pi}$ and $\alpha(x)=\mathrm{e}^{-x^{2}}$. We designate by $r\left(x^{\prime}, x, \tau_{0}\right)$ and $q\left(x^{\prime}, x, \tau_{0}\right)$ correspondingly the probabilities that a photon of frequency $x$ incident on an atmosphere of optical thickness $\tau_{0}$ (measured in the center of the line) will be reflected (transmitted) as a photon of frequency $x^{\prime}$. Adding an infinitesimal layer $\Delta$ and letting $\Delta \rightarrow 0$ yields

$$
\begin{aligned}
\frac{\mathrm{d} q}{\mathrm{~d} \tau_{0}} & =-\alpha(x) q\left(x^{\prime}, x, \tau_{0}\right) \\
& +\frac{\lambda\left(\tau_{0}\right)}{2} \int_{-\infty}^{\infty} q\left(x^{\prime}, x^{\prime \prime}, \tau_{0}\right) R\left(x^{\prime \prime}, x\right) \mathrm{d} x^{\prime \prime}+\frac{\lambda\left(\tau_{0}\right)}{2} \\
& \times \int_{-\infty}^{\infty} q\left(x^{\prime}, x^{\prime \prime}, \tau_{0}\right) \mathrm{d} x^{\prime \prime} \int_{-\infty}^{\infty} R\left(x^{\prime \prime}, x^{\prime \prime \prime}\right) r\left(x^{\prime \prime \prime}, x, \tau_{0}\right) \mathrm{d} x^{\prime \prime \prime} \\
\frac{\mathrm{d} r}{\mathrm{~d} \tau_{0}} & =-\left[\alpha(x)+\alpha\left(x^{\prime}\right)\right] r\left(x^{\prime}, x, \tau_{0}\right)+\frac{\lambda\left(\tau_{0}\right)}{2} R\left(x^{\prime}, x\right) \\
& +\frac{\lambda\left(\tau_{0}\right)}{2} \int_{-\infty}^{\infty} r\left(x^{\prime}, x^{\prime \prime}, \tau_{0}\right) R\left(x^{\prime \prime}, x\right) \mathrm{d} x^{\prime \prime} \\
& +\frac{\lambda\left(\tau_{0}\right)}{2} \int_{-\infty}^{\infty} R\left(x^{\prime}, x^{\prime \prime}\right) r\left(x^{\prime \prime}, x, \tau_{0}\right) \mathrm{d} x^{\prime \prime}+\frac{\lambda\left(\tau_{0}\right)}{2} \\
& \times \int_{-\infty}^{\infty} r\left(x^{\prime}, x^{\prime \prime}, \tau_{0}\right) \mathrm{d} x^{\prime \prime} \int_{-\infty}^{\infty} R\left(x^{\prime \prime}, x^{\prime \prime \prime}\right) r\left(x^{\prime \prime \prime}, x, \tau_{0}\right) \mathrm{d} x^{\prime \prime \prime} .
\end{aligned}
$$

As the initial conditions, we have $q\left(x^{\prime}, x, 0\right)=\delta\left(x-x^{\prime}\right)$ (where $\delta$ is the Dirac delta-function) and $r\left(x^{\prime}, x, 0\right)=0$, respectively. Note in passing that the same procedure applied to the transmittance, $\bar{q}\left(x^{\prime}, x, \tau_{0}\right)$, of a photon incident on the boundary 0 yields

$$
\begin{aligned}
\frac{\mathrm{d} \bar{q}}{\mathrm{~d} \tau_{0}} & =-\alpha(x) q\left(x^{\prime}, x, \tau_{0}\right) \\
& +\frac{\lambda\left(\tau_{0}\right)}{2} \int_{-\infty}^{\infty} R\left(x^{\prime}, x^{\prime \prime}\right) \bar{q}\left(x^{\prime \prime}, x, \tau_{0}\right) \mathrm{d} x^{\prime \prime}+\frac{\lambda\left(\tau_{0}\right)}{2} \\
& \times \int_{-\infty}^{\infty} r\left(x^{\prime}, x^{\prime \prime}, \tau_{0}\right) \mathrm{d} x^{\prime \prime} \int_{-\infty}^{\infty} R\left(x^{\prime \prime}, x^{\prime \prime \prime}\right) \bar{q}\left(x^{\prime \prime \prime}, x, \tau_{0}\right) \mathrm{d} x^{\prime \prime \prime},(44)
\end{aligned}
$$

subject to $\bar{q}\left(x^{\prime}, x, 0\right)=\delta\left(x-x^{\prime}\right)$. It is easy to conclude by comparing Eqs. (42) and (44) that the generalized form of the polarity property reads $\bar{q}\left(x^{\prime}, x, \tau_{0}\right)=q\left(x, x^{\prime}, \tau_{0}\right)$. At the same time, Eq. (40) implies that $r\left(x^{\prime}, x, \tau_{0}\right)=r\left(x, x^{\prime}, \tau_{0}\right)$ which is the case for a homogeneous atmosphere. Similar properties for the directions of the photon motion were established by Sobolev (1972) in considering the coherent scattering in a 3D-atmosphere.

Turning again to Eqs. (42) and (43), we replace the integrals over frequency with the Hermite-Gaussian quadrature sum. This quadrature formula is the best in some sense when the redistribution over frequency is Dopplerian, i.e., is due only to Doppler shifts. Choosing the zeros of the Hermite polynomials as the frequency grid, we may write the discrete analogs of Eqs. (42) and (43)

$$
\begin{aligned}
\frac{\mathrm{d} q_{i j}}{\mathrm{~d} \tau_{0}}= & -q_{i k}\left(\tau_{0}\right) \alpha_{k j}+\lambda\left(\tau_{0}\right) D_{k} q_{i k}\left(\tau_{0}\right) R_{k j} \\
& +2 \lambda\left(\tau_{0}\right) D_{k} D_{m} q_{i k}\left(\tau_{0}\right) R_{k m} r_{m j}\left(\tau_{0}\right), \\
\frac{\mathrm{d} r_{i j}}{\mathrm{~d} \tau_{0}}= & -\left[\alpha_{i k} r_{k j}\left(\tau_{0}\right)+r_{i k}\left(\tau_{0}\right) \alpha_{k j}\right]+\frac{1}{2} \lambda\left(\tau_{0}\right) R_{i j} \\
& +\lambda\left(\tau_{0}\right) D_{k} R_{i k} r_{k j}\left(\tau_{0}\right)+\lambda\left(\tau_{0}\right) D_{k} r_{i k}\left(\tau_{0}\right) R_{k j} \\
& +2 \lambda\left(\tau_{0}\right) D_{k} D_{m} r_{i k}\left(\tau_{0}\right) R_{k m} r_{m j}\left(\tau_{0}\right),
\end{aligned}
$$

where $q_{i j}\left(\tau_{0}\right)=q\left(x_{i}, x_{j}, \tau_{0}\right), r_{i j}\left(\tau_{0}\right)=r\left(x_{i}, x_{j}, \tau_{0}\right), R_{i j}=$ $R\left(x_{i}, x_{j}\right), \alpha_{i j}=\alpha_{i} \delta_{i j}\left(\delta_{i j}\right.$ is the unit matrix, $\left.\alpha_{i}=\alpha\left(x_{i}\right)\right), D_{i}$ are the weights of the quadrature formula. As usual, the repeated index means summation over this index. We recall that, since the transmittance is a non-symmetrical function of the frequency arguments, the sequence of indices in $q_{i j}$ is important. In the above equations, we chose the second index to denote the frequency of the incident photon, while the first one characterizes the scattered photon.

The transformations we perform with Eqs. (45) and (46) are the matrix analogs of those used in deriving the scalar Eqs. (13) and (16). As a result, we obtain

$$
\begin{aligned}
\frac{\mathrm{d} P_{i j}}{\mathrm{~d} \tau_{0}}= & \alpha_{i k} P_{k j}\left(\tau_{0}\right) \\
& -\lambda\left(\tau_{0}\right) D_{k} R_{i k}\left[P_{k j}\left(\tau_{0}\right)+S_{k j}\left(\tau_{0}\right)\right],
\end{aligned}
$$




$$
\begin{aligned}
\frac{\mathrm{d} S_{i j}}{\mathrm{~d} \tau_{0}}= & -\alpha_{i k} S_{k j}\left(\tau_{0}\right) \\
& +\lambda\left(\tau_{0}\right) D_{k} R_{i k}\left[P_{k j}\left(\tau_{0}\right)+S_{k j}\left(\tau_{0}\right)\right],
\end{aligned}
$$

where $P_{i j}\left(\tau_{0}\right)=q_{i j}^{-1}\left(\tau_{0}\right)$ and $S_{i j}\left(\tau_{0}\right)=2 D_{k} r_{i k}\left(\tau_{0}\right) q_{k j}^{-1}\left(\tau_{0}\right)$. Equations (47) and (48) are solved by using the initial conditions $P_{i j}(0)=\delta_{i j}, S_{i j}(0)=0$. Generalization of the proper equations for the left- and right-directed intensities, $U$ and $V$, within the atmosphere (see Eqs. (B1)) is straightforward:

$q\left(x^{\prime}, x, \tau_{0}\right)=\int_{-\infty}^{\infty} q\left(x^{\prime}, x^{\prime \prime}, \tau\right) U\left(\tau, x^{\prime \prime}, x, \tau_{0}\right) \mathrm{d} x^{\prime \prime}$,

$V\left(x^{\prime}, x, \tau_{0}\right)=\int_{-\infty}^{\infty} r\left(x^{\prime}, x^{\prime \prime}, \tau\right) U\left(\tau, x^{\prime \prime}, x, \tau_{0}\right) \mathrm{d} x^{\prime \prime}$.

In the vector-matrix form the required intensities are found from

$$
\begin{aligned}
& U_{i j}\left(\tau, \tau_{0}\right)=2 D_{k} P_{i k}(\tau) q_{k j}\left(\tau_{0}\right), \\
& V_{i j}\left(\tau, \tau_{0}\right)=2 D_{k} S_{i k}(\tau) q_{k j}\left(\tau_{0}\right) .
\end{aligned}
$$

We see that the main features inherent to the scalar case remain in the frequency-dependent formulation of the sourcefree problem. Separation of arguments in Eqs. (51) and (52) allows us to determine the internal radiation field in either of two possible ways. One may confine oneself to solving the set of Eqs. (45) and (46) with subsequent inversion of the matrix $q_{i j}$ to obtain $P_{i j}$ by one of the standard methods. Due to the diagonal-dominating character of the matrix $q_{i j}$, the Jacobi method seems to be better suited for this purpose. Another way is to solve the initial-value problem for the linear Eqs. (47) and (48). In both cases the internal radiation field is found simply by multiplying the proper matrices in accordance with Eqs. (51) and (52). Thus, as in the scalar formulation, the problem of diffuse reflection and transmission is reducible to solution of the initial-value problems which yield the requisite quantities for a set of atmospheres with different optical thickness.

Let us turn now to the frequency-dependent problem of the radiation transfer in an atmosphere with energy sources distributed inside it. The scalar case of this problem is considered in Appendix D, where we derived Eqs. (D.8) and (D.9) to determine the emergent intensities. Simple reasoning allows us to generalize these to write

$$
\begin{aligned}
\frac{\mathrm{d} I_{1}}{\mathrm{~d} \tau_{0}}= & -\alpha(x) I_{1}\left(x, \tau_{0}\right) \\
& +\frac{\lambda\left(\tau_{0}\right)}{2} \int_{-\infty}^{\infty} R\left(x, x^{\prime}\right) I_{1}\left(x^{\prime}, \tau_{0}\right) \mathrm{d} x^{\prime} \\
& +\frac{1}{2}\left[B\left(x, \tau_{0}\right)+\int_{-\infty}^{\infty} r\left(x, x^{\prime}, \tau_{0}\right) B\left(x^{\prime}, \tau_{0}\right) \mathrm{d} x^{\prime}\right],
\end{aligned}
$$
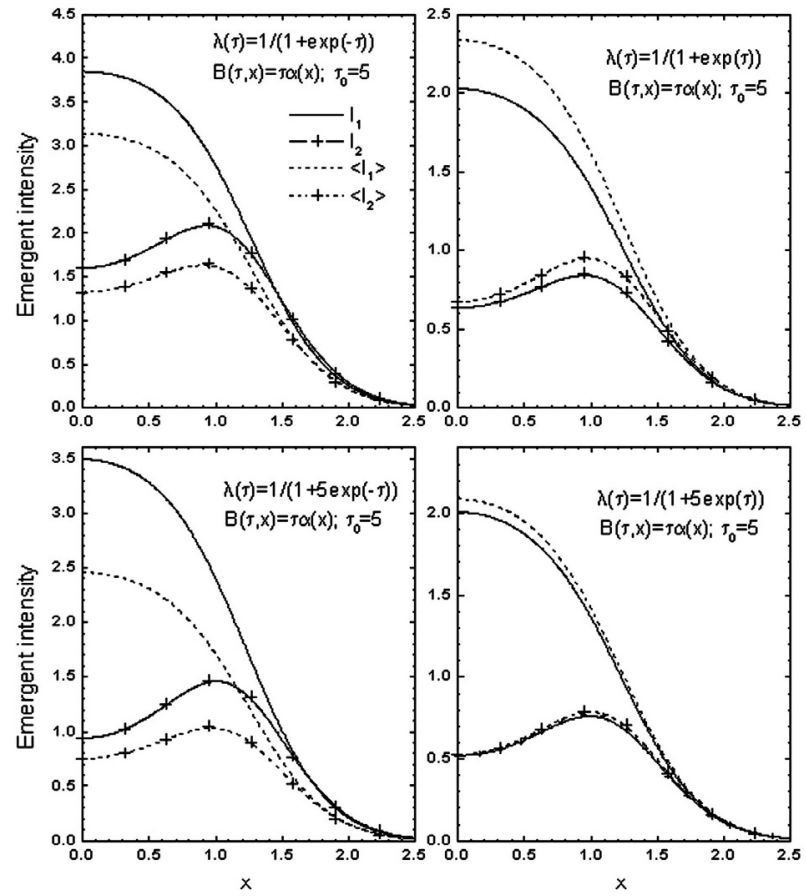

Fig. 4. Profiles of spectral lines formed in two kinds of atmosphere for $\tau_{0}=5$ and $a=1 ; 5$. Solid lines represent the profiles observed from the boundaries $\tau_{0}$ and 0 (crossed line). Accordingly, the dotted lines correspond to the profiles relevant to the atmosphere with singlescattering albedo averaged over thickness.

$$
\begin{aligned}
\frac{\mathrm{d} I_{2}}{\mathrm{~d} \tau_{0}}= & \frac{\lambda\left(\tau_{0}\right)}{2} \\
& \times \int_{-\infty}^{\infty} q\left(x, x^{\prime}, \tau_{0}\right) \mathrm{d} x^{\prime} \int_{-\infty}^{\infty} R\left(x^{\prime}, x^{\prime \prime}\right) I_{1}\left(x^{\prime \prime}, \tau_{0}\right) \mathrm{d} x^{\prime \prime} \\
& +\frac{1}{2} \int_{-\infty}^{\infty} q\left(x, x^{\prime}, \tau_{0}\right) B\left(x^{\prime}, \tau_{0}\right) \mathrm{d} x^{\prime}
\end{aligned}
$$

where $B\left(x, \tau_{0}\right)$ is the power of the energy sources.

The non-normalized profiles of the spectral lines formed in atmospheres of the first and second kinds with $\tau_{0}=5$ are illustrated in Fig. 4. The profiles observed from both sides of the atmosphere are plotted for the linearly increasing function of $B(x, \tau)=\tau \alpha(x)$ and two values of the parameter $a=(1,5)$. For simplicity, we considered the case of complete redistribution over frequencies with Dopplerian profiles for the absorption coefficient in the line. The profiles computed for the average value of the single-scattering albedo are also presented (dotted lines).

The main features of these profiles may be summarized as follows. It is seen that, independent of the depth-variation of $\lambda$, the double-peaked profiles are observed only when the relatively faint layers of the atmosphere are projected onto bright ones. As could be expected, the greater the difference between the real and averaged profiles, the more efficient the scattering. Note also that the character of this departure depends on the depth variation of the single-scattering albedo.

In conclusion, we briefly consider the radiation field within the atmosphere with distributed sources. Now the requisite 
intensities $I^{ \pm}$are functions of frequency, and in place of Eqs. (D.12) and (D.13) we have

$$
\begin{aligned}
I_{2}\left(x, \tau_{0}\right)= & \int_{-\infty}^{\infty} q\left(x, x^{\prime}, \tau\right) I^{+}\left(\tau, x^{\prime}, \tau_{0}\right) \mathrm{d} x^{\prime} \\
& +I_{2}(x, \tau), \\
I^{-}\left(\tau, x, \tau_{0}\right)= & \int_{-\infty}^{\infty} r\left(x, x^{\prime}, \tau\right) I^{+}\left(\tau, x^{\prime}, \tau_{0}\right) \mathrm{d} x^{\prime} \\
& +I_{1}(x, \tau) .
\end{aligned}
$$

Written in the vector-matrix form, these imply

$$
\begin{aligned}
& I_{k}^{+}\left(\tau, \tau_{0}\right)=2 D_{i} P_{k i}\left(\tau_{0}\right)\left[I_{2 i}\left(\tau_{0}\right)-I_{2 i}(\tau)\right], \\
& I_{k}^{-}\left(\tau, \tau_{0}\right)=2 D_{i} r_{k i}(\tau) I_{i}^{+}\left(\tau, \tau_{0}\right)+I_{1 k}(\tau) .
\end{aligned}
$$

Thus, as in the source-free problem, the knowledge of quantities $r_{i j}, q_{i j}$ and $P_{i j}=q_{i j}^{-1}$ is sufficient to find the intensities $I^{ \pm}$ simply by multiplying the appropriate matrices.

\section{Concluding remarks}

Extending Ambartsumian's method of addition of layers to handle the transfer of radiation in plane-parallel inhomogeneous atmospheres, we treated several standard and frequently encountered problems of astrophysical interest. It is shown that all of these are reducible to the initial-value problems for the linear differential equations of the first order. This allows one to avoid the difficulties specific to the classical approach which leads to the two-point boundary-value problems with conditions of Sturm's type. The method proposed in this work assumes that one starts with determining the reflection and transmission capacities of the atmosphere. Knowledge of the quantities $r$ and $q$ (or $r_{i k}, q_{i k}$ and $q_{i k}^{-1}$, in the matrix case) makes it easy to find some other characteristics of the radiation diffusion process. This primarily concerns the internal radiation field whose determination does not need any integration. The method possesses some other advantages and may be generalized in various directions. For instance, with slightly more effort one may reformulate the reasoning in treating the frequency-dependent problem to handle the three-dimensional version of the considered problems. On the other hand, although only the single-scattering albedo and the power of internal energy sources were assumed to vary in an atmosphere, the approach is applicable to a class of transfer problems in which other characteristics of the elementary act of scattering (redistribution function, indicatrix, the parameters of the polarized radiation, etc.) are allowed to vary with depth. The linearity of equations for the functions $P$ and $S$ enables one to use the well-developed analytical and numerical methods for solving the Cauchy problems for a set of linear differential equations. Note also that the method of addition of layers makes it possible to evaluate the required quantities simultaneously for a family of atmospheres of different optical thickness. One of the main results of the work is the recursion formulas, which make it easy to find the requisite intensities for a multicomponent atmosphere. Further extension of this result will be of great importance from the point of view of astrophysical applications. In considering the transfer problems with partial redistribution of radiation over frequencies, it is more efficient to use bilinear expansions of the proper redistribution functions (see Hummer 1962; Nikoghossian 1977) in turning to the discrete analogs of the equations under consideration.

Acknowledgements. The author is thankful to Acad. D. Sedrakian for useful discussions.

\section{References}

Abhyankar, K. D., \& Fymat, A. L. 1969, ApJ, 158, 325

Abhyankar, K. D., \& Fymat, A. L. 1970, ApJ, 159, 1009

Ambartsumian, V. A. 1943, Sov. Doklady, 38, 257

Ambartsumian, V. A. 1944, Proc. Acad. Sci. ArmSSR, No. 1-2

Ambartsumian, V. A. 1948, Doklady ArmSSR, 8, 101

Ambartsumian, V. A. 1960, Scientific Works, 1, Izd. Acad. Nauk Arm. SSR, Yerevan (in Russian)

Bakhvalov, N. S. 1973, Numerical Methods, 1 (Moscow: Nauka) (in Russian)

Barletti, L., \& Cecchi-Pestellini, C. 2001, New Astron., 6, 165

Bellman, R. E., \& Kalaba, R. E. 1956, Proc. Nat. Acad. Sci. USA, 42, 629

Bellman, R. E., \& Kalaba, R. E. 1960, J. Math. Phys., 1, 280

Berezin, L. S., \& Zhidkhov, N. P. 1965, Computing Methods, (Reading, Mass.: Addison - Wesley)

Busbridge, I. W. 1961, ApJ, 133, 198

Chandrasekhar, S. 1960, Radiative Transfer, Dever, New York

Cecchi-Pestellini, C., \& Barletti, L. 2001, New Astron., 6, 151

Collatz, L. 1960, The Numerical Treatment of Differential Equations (Berlin: Springer-Verlag)

Gu, Y., Lindsey, C., \& Jefferies, J. T. 1995, ApJ, 450, 318

Gu, Y., Lindsey, C., Jefferies, J. T., \& Avrett, E. H. 1997, ApJ, 484, 960

Jefferies, J. T., \& Lindsey, C. 1988, ApJ, 335, 372

Juvela, M., \& Padoan, P. 2003, A\&A, 397, 201

Hegmann, M., \& Kegel, W. H. 2003, MNRAS, 342, 453

Hummer, D. G. 1962, MNRAS, 125, 21

Lindsey, C., \& Jefferies, J. T. 1990, ApJ, 349, 286

Meinkohn, E., \& Richling, S. 2002, A\&A, 392, 827

Nikoghossian, A. G. 1977, Sov. Doklady, 235, 786

Nikoghossian, A. G. 1984a, Afz, 21, 323

Nikoghossian, A. G. 1984b, Afz, 21, 579

Nikoghossian, A. G., Pojoga, S., \& Mouradian, Z. 1997, A\&A, 325 813

Nikoghossian, A. G., Pojoga, S., \& Mouradian, Z. 1999, A\&A, 342, 785

Nikoghossian, A. G., \& Mouradian, Z. 2000, A\&A, 360, 1095

Peraiah, A. 1984, in Methods in Radiative Transfer, ed. W. Kalkofen (Cambridge University Press)

Pomraning, G. C. 1988, JQSRT, 40, 479

Preisendorfer, R. W. 1958, Proc. Nat. Acad. Sci. USA, 44, 323

Preisendorfer, R. W. 1976, Hydrologic Optics, IV, US Dept. of Commerce, Honolulu, Hawaii

Sobolev, V. V. 1963, A Treatise on Radiative Transfer (Princeton: van Nostrand)

Sobolev, V. V. 1972, Scattering of the light in atmospheres of planets (Moscow: Nauka) (in Russian)

Sobolev, V. V. 1974, AZh, 51, 50 
A. G. Nikoghossian: Radiative transfer in 1D inhomogeneous atmospheres, Online Material $p 1$

\section{Online Material}




\section{Appendix A: Examples of analytical solutions}

(a) Let $\lambda\left(\tau_{0}\right)=\left[1+a \mathrm{e}^{-\tau_{0}}\right]^{-1}$, where $a$ is a positive parameter. Then $1-\lambda-\left(\lambda^{\prime} / \lambda\right)=0$, and the order of Eq. (22) can be reduced. After some simple algebra we finally obtain

$q\left(\tau_{0}\right)=\left[1+\left(a+\frac{1}{2}\right) \ln \frac{\mathrm{e}^{\tau_{0}}+a}{1+a}\right]^{-1}$,

$r\left(\tau_{0}\right)=1+2 a \mathrm{e}^{-\tau_{0}}-(1+2 a) q\left(\tau_{0}\right)$.

The validity of these results in limiting cases is easy to check. We see that, as $\tau_{0} \rightarrow \infty, q\left(\tau_{0}\right) \rightarrow 0$, and $r\left(\tau_{0}\right) \rightarrow 1$. If $a=0$, we have $\lambda=1$, i.e., a purely scattering atmosphere, and Eqs. (A.1) and (A.2) lead to the relations $q\left(\tau_{0}\right)=2 /\left(2+\tau_{0}\right)$, $r\left(\tau_{0}\right)=1-q\left(\tau_{0}\right)$, well known in the theory of the radiative transfer in homogeneous atmospheres. As $a \rightarrow \infty$, the atmosphere approaches the LTE limit, for which $q\left(\tau_{0}\right)=\mathrm{e}^{-\tau_{0}}$, $r\left(\tau_{0}\right)=0$.

The last characteristic of the considered atmosphere is $\bar{r}\left(\tau_{0}\right)$ which is given by Eq. (11). The net result is

$\bar{r}\left(\tau_{0}\right)=\frac{1-q\left(\tau_{0}\right)}{1+2 a}$,

where $q\left(\tau_{0}\right)$ is given by Eq. (A.1).

(b) Let us now assume that $\lambda\left(\tau_{0}\right)=\left[1+a \mathrm{e}^{\tau_{0}}\right]^{-1}$, where, again, $a$ is a certain positive parameter. Atmospheres with this behavior of $\lambda\left(\tau_{0}\right)$ will be referred hereafter to as atmospheres of the second kind, as distinguished from those in example (a) which are atmospheres of the first kind. It is easy to see that $1-$ $\lambda+\left(\lambda^{\prime} / \lambda\right)=0$, and now Eq. (23) is simplified and resolves itself to a differential equation of the first order. The final result is

$$
\begin{aligned}
& q\left(\tau_{0}\right)=\left[1+\left(a \mathrm{e}^{\tau_{0}}+\frac{1}{2}\right) \ln \frac{1+a}{\mathrm{e}^{-\tau_{0}}+a}\right]^{-1}, \\
& r\left(\tau_{0}\right)=\frac{1-q\left(\tau_{0}\right)}{1+2 a \mathrm{e}^{\tau_{0}}} .
\end{aligned}
$$

For $\bar{r}\left(\tau_{0}\right)$, Eq. (11) yields

$\bar{r}\left(\tau_{0}\right)=1+2 a-\left(1+2 a \mathrm{e}^{\tau_{0}}\right) q\left(\tau_{0}\right)$,

where $q\left(\tau_{0}\right)$ satisfies Eq. (A.4). Here again $q\left(\tau_{0}\right) \rightarrow 0$, as $\tau_{0} \rightarrow$ $\infty$, while, in contrast to the previous example, $r\left(\tau_{0}\right) \rightarrow 0$.

\section{Appendix B: Internal field of radiation}

Let us consider an atmosphere of a fixed optical thickness $\tau_{0}$ with a photon falling on the boundary $\tau_{0}$ (see Fig. B.1). We introduce the quantities $U\left(\tau, \tau_{0}\right)$ and $V\left(\tau, \tau_{0}\right)$ to denote the probabilities that the incident photon will be found (in general, as a result of multiple scattering) at depth $\tau$ moving respectively toward boundaries 0 and $\tau_{0}$. Taking into account the probability meaning of the reflection and transmission coefficients, we may write

$$
\begin{aligned}
& q\left(\tau_{0}\right)=q(\tau) U\left(\tau, \tau_{0}\right), \\
& V\left(\tau, \tau_{0}\right)=r(\tau) U\left(\tau, \tau_{0}\right)
\end{aligned}
$$

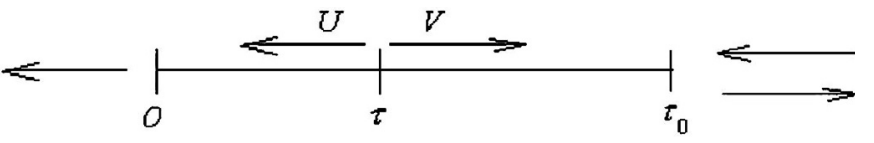

Fig. B.1. The radiative transfer process in an atmosphere of the optical thickness $\tau_{0}$.

The physical meaning of the second relation is apparent, while the first one is not so evident, as it may seem. It may be regarded as a partial manifestation of the well-known semi-group property of the $U$-function (see, e.g., Preisendorfer 1976). The rigorous proof of this relation we shall give below using the system of equations Eqs. (13) and (16).

Now we see that, once the reflectance and transmittance are found, the desired probabilities $U$ and $V$ are obtained directly from

$U\left(\tau, \tau_{0}\right)=\frac{q\left(\tau_{0}\right)}{q(\tau)}=q\left(\tau_{0}\right) P(\tau)$,
$V\left(\tau, \tau_{0}\right)=q\left(\tau_{0}\right) \frac{r(\tau)}{q(\tau)}=q\left(\tau_{0}\right) S(\tau)$.

These relations follow also from the standard equations of radiative transfer which, being expressed in terms of the $U$ - and $V$-functions, acquire the form

$\frac{\mathrm{d} U}{\mathrm{~d} \tau}=\left(1-\frac{\lambda(\tau)}{2}\right) U\left(\tau, \tau_{0}\right)-\frac{\lambda(\tau)}{2} V\left(\tau, \tau_{0}\right)$

$\frac{\mathrm{d} V}{\mathrm{~d} \tau}=\frac{\lambda(\tau)}{2} U\left(\tau, \tau_{0}\right)-\left(1-\frac{\lambda(\tau)}{2}\right) V\left(\tau, \tau_{0}\right)$

As initial conditions we can choose $U\left(0, \tau_{0}\right)=q\left(\tau_{0}\right)$, $V\left(0, \tau_{0}\right)=0$. Thus, we see that $U\left(\tau, \tau_{0}\right)$ and $V\left(\tau, \tau_{0}\right)$ (as functions of optical depth) satisfy the same system of differential equations Eqs. (13) and (16), as the above introduced quantities $P$ and $S$ dependent on optical thickness. Comparing the initial conditions of Eqs. (B.3), (B.4) and (13), (16) we arrive at Eqs. (B.2). This particularly implies that the depth-dependence of functions $U$ and $V$ also satisfy the second-order differential equations similar to Eqs. (22) and (23). Thus, the quantities $P$ and $S$ regarded as functions of $\tau$ describe to within a constant factor the depth-variation of the radiation fluxes in two mutually opposite directions.

The most important and somewhat unexpected result is the factorization of the functions $U\left(\tau, \tau_{0}\right), V\left(\tau, \tau_{0}\right)$. All co-factors are simply related to the reflectance and transmittance of the atmosphere determined in Sect. 3. This is one of the advantages of the approach we propose. Note that one may use Eqs. (A.1)-(A.4) to write down the explicit analytical expressions for the radiation intensities within atmospheres of the first and second kinds. In addition, Eqs. (B.2) make it possible to reveal some features inherent, in general, in the radiation transfer in inhomogeneous atmospheres. For instance, these equations imply that the radiation flux in both directions at a certain depth $\tau$ in atmospheres of different optical thickness is proportional to their opacity. On the other hand, the intensities at different depths, $\tau$, in the same atmosphere are inversely proportional to opacities $q(\tau)$ corresponding to these depths. 
A. G. Nikoghossian: Radiative transfer in 1D inhomogeneous atmospheres, Online Material $p 3$

Note in conclusion that an analogous consideration allows us to find the internal radiation field if the atmosphere is illuminated from the boundary 0 .

\section{Appendix C: Mean number of scatterings}

Let us turn to Fig. 1 and treat again an inhomogeneous atmosphere, consisting of two parts with optical thickness $\tau_{1}$ and $\tau_{2}$, which is illuminated from the boundary $\tau_{0}=\tau_{1}+\tau_{2}$. The incident photons undergo true absorption in the atmosphere or escape it, in general, after multiple scattering. We introduce notations $N_{r}, N_{q}$ to denote the mean number of scatterings undergone by the reflected and transmitted photons, respectively. As above, the proper quantities relating to the photons incident on the opposite boundary will be marked by bars. Equations (1) and (2) show the elementary events that result in the reflection and transmission processes characterized by the coefficients $r\left(\tau_{1}+\tau_{2}\right)$ and $q\left(\tau_{1}+\tau_{2}\right)$. Each of these events occurs, on an average, as a result of a certain number of scatterings. Consequently Eqs. (1) and (2) can be used to write similar equations for the expected values of the mean numbers of scattering $q N_{q}$ and $r N_{r}$. For instance, for transmitted photons we may write

$$
\begin{aligned}
q\left(\tau_{1}+\tau_{2}\right) & N_{q}\left(\tau_{1}+\tau_{2}\right)=q\left(\tau_{1}\right) q^{*}\left(\tau_{2}\right)\left[N_{q}\left(\tau_{1}\right)+N_{q}^{*}\left(\tau_{2}\right)\right] \\
& +q\left(\tau_{1}\right) r\left(\tau_{1}\right) \bar{r}^{*}\left(\tau_{2}\right) q^{*}\left(\tau_{2}\right) \\
& \times\left[N_{q}\left(\tau_{1}\right)+N_{q}^{*}\left(\tau_{2}\right)+N_{r}\left(\tau_{1}\right)+\bar{N}_{r}^{*}\left(\tau_{2}\right)\right]+\ldots
\end{aligned}
$$

We recall that the asterisks are used to denote the difference in the domains of variation of the function $\lambda(\tau)$. To derive the necessary differential equations, we replace, as before, $\tau_{2}$ by infinitesimal $\Delta$ and pass to the limit when $\Delta \rightarrow 0$. Then replacing $\tau_{1}$ by $\tau_{0}$ we obtain

$$
\begin{aligned}
\frac{\mathrm{d}\left(q N_{q}\right)}{\mathrm{d} \tau_{0}}= & -A\left(\tau_{0}\right) q\left(\tau_{0}\right) N_{q}\left(\tau_{0}\right) \\
& +\frac{\lambda\left(\tau_{0}\right)}{2} q\left(\tau_{0}\right)\left[1+r\left(\tau_{0}\right)\left(1+N_{r}\left(\tau_{0}\right)\right)\right] .
\end{aligned}
$$

Similarly for the reflected photons we obtain

$$
\begin{aligned}
\frac{\mathrm{d}\left(r N_{r}\right)}{\mathrm{d} \tau_{0}}= & -2 A\left(\tau_{0}\right) r\left(\tau_{0}\right) N_{r}\left(\tau_{0}\right) \\
& +\frac{\lambda\left(\tau_{0}\right)}{2}\left[1+r\left(\tau_{0}\right)\right]^{2} .
\end{aligned}
$$

At $\tau_{0}=0$, the quantities $r N_{r}, q N_{q}$ are equal to 0 . It is easy to check that Eqs. (C.2) and (C.3) are obtainable from the proper equations for transmittance and reflectance (Eqs. (8) and (9)) by formal differentiation with respect to $\lambda$, regarding the latter as a parameter. Such a procedure for evaluating the mean number of scatterings was proposed by Ambartsumian (1948) (see also Ambartsumian 1960) for homogeneous atmospheres. Now we see that it remains valid when inhomogeneities are allowed. It should be emphasized, however, that this conclusion concerns merely the photons escaping the atmosphere but not those thermalized in it (see Nikoghossian 1984a,b).

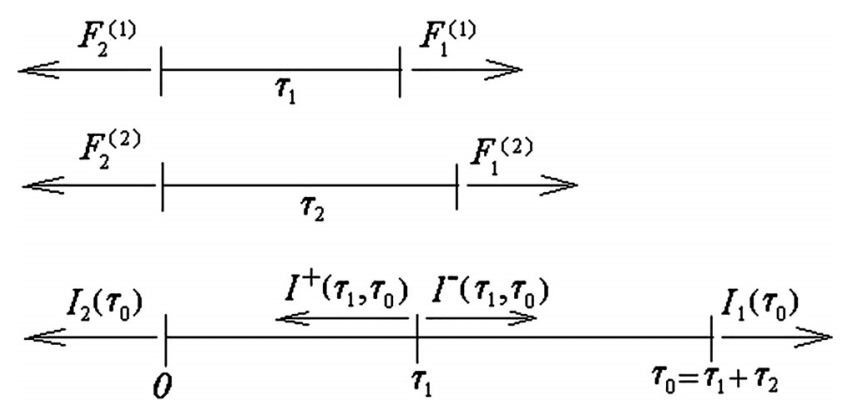

Fig. D.1. Addition of layers for scattering with distributed sources.

Taking into account Eq. (8) for the transmission coefficient, solutions of Eqs. (C.2) and (C.3) may be presented in the integral form

$N_{q}\left(\tau_{0}\right)=\frac{1}{2} \int_{0}^{\tau_{0}} \lambda(t)\left\{1+r(t)\left[1+N_{r}(t)\right]\right\} \mathrm{d} t$
$N_{r}\left(\tau_{0}\right)=\frac{q^{2}\left(\tau_{0}\right)}{2 r\left(\tau_{0}\right)} \int_{0}^{\tau_{0}} \lambda(t)\left[1+r^{2}(t)\right] \frac{\mathrm{d} t}{q^{2}(t)}$

Differential equations similar to Eqs. (C.2) and (C.3) may be readily derived for the case when the atmosphere is illuminated from the boundary 0 . Here we restrict this by presenting the final result

$\frac{\mathrm{d}\left(\bar{r} \bar{N}_{r}\right)}{\mathrm{d} \tau_{0}}=\frac{\lambda\left(\tau_{0}\right)}{2} q\left(\tau_{0}\right)\left[q\left(\tau_{0}\right)+2 N_{q}\left(\tau_{0}\right)\right]$,

subject to $\bar{r} \bar{N}_{r}(0)=0$. We note that $q N_{q}=\bar{q} \bar{N}_{q}$, so that Eq. (C.2) holds in this case as well.

\section{Appendix D: Internal sources of energy}

Turning to the problem of scattering in an atmosphere with distributed sources let us introduce the function $B(\tau)$ to describe the variation of the power of the internal energy sources with depth. As in the problem of diffuse reflection and transmission, we begin with determining the intensities of the emergent radiation.

Consider a radiating atmosphere of optical thickness $\tau_{0}$, in which both the power of internal sources and the singlescattering albedo are allowed to vary with optical depth. To find the emergent intensities $I_{1}\left(\tau_{0}\right)=I^{-}\left(\tau_{0}, \tau_{0}\right)$ and $I_{2}\left(\tau_{0}\right)=$ $I^{+}\left(0, \tau_{0}\right)$, let us mentally divide the atmosphere into two parts of optical thickness $\tau_{1}$ and $\tau_{2}\left(\tau_{0}=\tau_{1}+\tau_{2}\right)$ (see Fig. D.1). Suppose that each of these parts taken alone radiates to both sides the intensities $F_{1}^{(1)}, F_{2}^{(1)}$ and $F_{1}^{(2)}, F_{2}^{(2)}$. As a result of their interaction in the composite atmosphere, we observe at depth $\tau_{1}$ the intensities $I^{+}\left(\tau_{1}, \tau_{0}\right)$ and $I^{-}\left(\tau_{1}, \tau_{0}\right)$. Making use of Fig. D.1, for the introduced quantities, one may write a series of simple, physically intelligible relations

$I_{1}\left(\tau_{0}\right)=I^{-}\left(\tau_{1}, \tau_{0}\right) q^{*}\left(\tau_{2}\right)+F_{1}^{(2)}$

$I_{2}\left(\tau_{0}\right)=I^{+}\left(\tau_{1}, \tau_{0}\right) q\left(\tau_{1}\right)+F_{2}^{(1)}$, 
A. G. Nikoghossian: Radiative transfer in 1D inhomogeneous atmospheres, Online Material p 4

$I^{-}\left(\tau_{1}, \tau_{0}\right)=I^{+}\left(\tau_{1}, \tau_{0}\right) r\left(\tau_{1}\right)+F_{1}^{(1)}$,

$I^{+}\left(\tau_{1}, \tau_{0}\right)=I^{-}\left(\tau_{1}, \tau_{0}\right) \bar{r}^{*}\left(\tau_{2}\right)+F_{2}^{(2)}$.

Taken together, Eqs. (D.3) and (D.4) yield

$$
\begin{aligned}
I^{-}\left(\tau_{1}, \tau_{0}\right)= & \frac{r\left(\tau_{1}\right)}{1-r\left(\tau_{1}\right) \bar{r}^{*}\left(\tau_{2}\right)} F_{2}^{(2)} \\
& +\frac{1}{1-r\left(\tau_{1}\right) \bar{r}^{*}\left(\tau_{2}\right)} F_{1}^{(1)},
\end{aligned}
$$

which incorporated into Eq. (D.1) allows us to write

$$
\begin{aligned}
I_{1}\left(\tau_{0}\right)= & \frac{q\left(\tau_{2}\right)}{1-r\left(\tau_{1}\right) \bar{r}^{*}\left(\tau_{2}\right)} \\
& \times\left[r\left(\tau_{1}\right) F_{2}^{(2)}+F_{1}^{(1)}\right]+F_{1}^{(2)} .
\end{aligned}
$$

Proceeding in a similar way, from Eq. (D.2) we may derive

$$
\begin{aligned}
I_{2}\left(\tau_{0}\right)= & \frac{q\left(\tau_{1}\right)}{1-r\left(\tau_{1}\right) \bar{r}^{*}\left(\tau_{2}\right)} \\
& \times\left[\bar{r}\left(\tau_{2}\right) F_{1}^{(1)}+F_{2}^{(2)}\right]+F_{2}^{(1)} .
\end{aligned}
$$

Suppose now that $\tau_{2}$ is infinitesimal and equals $\Delta$. With an accuracy to the first order of $\Delta$, the infinitesimal layer can be regarded as homogeneous and isothermal so that $F_{1}^{(2)}=F_{2}^{(2)}=$ (1/2) $B \Delta$. Then passing to the limit as $\Delta \rightarrow 0$, from Eqs. (D.6) and (D.7) we arrive at the following differential equations

$\frac{\mathrm{d} I_{1}}{\mathrm{~d} \tau_{0}}=-A\left(\tau_{0}\right) I_{1}\left(\tau_{0}\right)+\frac{1}{2}\left[1+r\left(\tau_{0}\right)\right] B\left(\tau_{0}\right)$

$\frac{\mathrm{d} I_{2}}{\mathrm{~d} \tau_{0}}=\frac{1}{2} q\left(\tau_{0}\right)\left[\lambda\left(\tau_{0}\right) I_{1}\left(\tau_{0}\right)+B\left(\tau_{0}\right)\right]$,

subject to $I_{1}(0)=I_{2}(0)=0$.
This implies the integral representations for the requisite intensities

$I_{1}\left(\tau_{0}\right)=\frac{1}{2} q\left(\tau_{0}\right) \int_{0}^{\tau_{0}}[1+r(t)] B(t) \frac{\mathrm{d} t}{q(t)}, \mathrm{d} t$.

$I_{2}\left(\tau_{0}\right)=\frac{1}{2} \int_{0}^{\tau_{0}} q(t)\left[\lambda(t) I_{1}(t)+B(t)\right] \mathrm{d} t$.

In writing Eq. (D.10) we used Eq. (8). The expression within the integral in Eq. (D.10) may be interpreted as the product of the power of the energy sources and the photon exit probability. As for Eq. (D.11), it merely states that the emergent intensity consists of two parts: the first is due to the opposite directed radiation that is scattered and penetrates to the surface from each element of optical depth, while the second results directly from internal sources of energy.

Thus, the required intensities are explicitly expressed in terms of reflectance and transmittance of an atmosphere. In the case of the pure absorbing $\left(\lambda\left(\tau_{0}\right) \equiv 0\right)$ and the pure scattering atmospheres $\left(\lambda\left(\tau_{0}\right) \equiv 1\right)$ we are led for $B\left(\tau_{0}\right)=$ const. to the well-known results: $I_{1}\left(\tau_{0}\right)=I_{2}\left(\tau_{0}\right)=(B / 2)\left[1-\exp \left(-\tau_{0}\right)\right]$ and $I_{1}\left(\tau_{0}\right)=I_{2}\left(\tau_{0}\right)=B \tau_{0} / 2$, respectively.

By analogy to the source-free problem, the emergent intensities having been determined, the internal radiation field is found in a simple way not demanding any additional integration. Indeed, using Eqs. (D.2) and (D.3) we obtain

$I^{+}\left(\tau, \tau_{0}\right)=\left[I_{2}\left(\tau_{0}\right)-I_{2}(\tau)\right] / q(\tau)$,

$I^{-}\left(\tau, \tau_{0}\right)=r(\tau) I^{+}\left(\tau, \tau_{0}\right)+I_{1}(\tau)$. 